Research Article

\title{
Circulation of Dengue Virus Serotypes in the City of Makkah, Saudi Arabia, as Determined by Reverse Transcription Polymerase Chain Reaction
}

\author{
Sameer R. Organji, ${ }^{1}$ Hussein H. Abulreesh, ${ }^{1}$ and Gamal E. H. Osman ${ }^{1,2}$ \\ ${ }^{1}$ Department of Biology, Faculty of Applied Science, Umm Al-Qura University, Makkah 21955, Saudi Arabia \\ ${ }^{2}$ Microbial Genetics Department, Agricultural Genetic Engineering Research Institute (AGERI), ARC, Giza 12619, Egypt \\ Correspondence should be addressed to Hussein H. Abulreesh; hhabulreesh@uqu.edu.sa
}

Received 29 November 2016; Revised 17 January 2017; Accepted 29 January 2017; Published 19 February 2017

Academic Editor: José Ramón Blanco

Copyright ( 12017 Sameer R. Organji et al. This is an open access article distributed under the Creative Commons Attribution License, which permits unrestricted use, distribution, and reproduction in any medium, provided the original work is properly cited.

\begin{abstract}
The present study was aimed to investigate the circulation of four dengue virus (DENV) serotypes in Makkah, Western Saudi Arabia. Blood samples were collected from 25 dengue fever-suspected patients and were subjected to molecular typing for DENV1, DENV-2, DENV-3, and DENV-4 serotypes of dengue virus, by reverse transcription polymerase chain reaction (RT-PCR), using six sets of primers. Of the 25 samples, only six samples (24\%) were found to be positive for dengue virus infection. The prevalence of DENV-1 was higher (50\% of DENV-positive samples), as compared to DENV-2 (33.3\%) and DENV-3 (16.6\%) serotypes. The fourth serotype, DENV-4, was not detected in any of the DENV-positive samples. Although Makkah is considered endemic to dengue fever, we observed low prevalence of dengue virus in the city, which may be attributed to various factors. Nonetheless, the results presented herein confirm the circulation of DENV serotypes in the Western region of Saudi Arabia. To the best of our knowledge, the current study so far is the first report demonstrating the prevalence of the DENV-1 serotype in the city Makkah, Saudi Arabia.
\end{abstract}

\section{Introduction}

Dengue fever/dengue hemorrhagic fever (DF/DHF) is one of the major reemerging diseases in the past decade that has been expanding its circulation in various geographic locations around the world. The virus infection is characterized by a spectrum of illnesses that ranges from mild febrile illness to the fatal hemorrhagic disease and the dengue shock syndrome (DSS) [1-3]. Dengue virus infection constitutes a major public health concern and is estimated to cause about 300 million cases in a year across the globe. About 3.97 billion people, who are living in the epidemic areas in the tropical and subtropical regions, are at risk of acquiring the viral infection $[2,4]$.

Dengue fever/dengue hemorrhagic fever and the dengue shock syndrome are the diseases that are caused by dengue virus (DENV) that belongs to the genus Flavivirus a member of the Flaviviridae family. There are four dengue virus serotypes DENV-1, DENV-2, DENV-3, and DENV-4 that have similar antigenic properties but are distinct from one another in terms of specificity to the anti-DENV antibodies [2-4]. Dengue virus infection is an arthropod vector-borne disease that is transmitted by different species of Aedes mosquito (Ae. aegypti; Ae. albopictus; Ae. polynesiensis). Ae. aegypti is considered to be the main vector because it is in proximity to human habitation $[1,2,4]$.

As reported by various epidemiological studies, the areas such as the South and Southeast Asia, South Africa, Africa, and the Arabian peninsula, which have a wide circulation of dengue viruses and/or their vectors, are considered to be dengue endemic areas $[1,2]$. The dengue virus circulation was also reported in the regions that are located at the $35^{\circ} \mathrm{N}$ latitude (e.g., Korea and Japan), where the temperature in the winter season goes below $10^{\circ} \mathrm{C}$ at which the survival of $A e$. aegypti becomes difficult $[5,6]$.

Saudi Arabia was considered to be a dengue-free country, until 1994, when the first dengue fever outbreak was reported in Jeddah that resulted in 289 confirmed cases of the viral 
infection [7]. The subsequent outbreaks occurred in different cities of the Western and Southwestern regions of Saudi Arabia, that is, Jeddah, Makkah, Aseer, and Jizan, in the years 1994 through 2010. By then the Western and Southwestern regions of Saudi Arabia were declared as the dengue endemic areas [8-13].

In the city of Makkah, the number of DF cases has increased since the first outbreak of the disease in 2004; however, only two reports are available, one published in 2008 [10] and the other published in 2009 [14], regarding the rise of DF cases and the prevalence of dengue virus serotypes in Makkah. As per the above-mentioned articles, DENV-2 and DENV-3 were the only serotypes detected in the cases of DF/ DHF/DSS in Makkah [10]; however in one study by Shahin et al. [14], none of the DENV serotypes was identified. Thus, in such a dearth of information on the circulation of DENV serotypes in Makkah, we aimed to analyze the prevalence of DENV serotypes in DF-diagnosed patients in the city of Makkah, Western Saudi Arabia.

\section{Methods}

2.1. Sample Collection and Preparation. Blood samples $(2.0 \mathrm{~mL})$ from 25 patients presenting dengue-like clinical manifestations were collected in the commercially available EDTA anticoagulant tubes. The red blood cells were separated from the plasma by centrifugation at $3000 \mathrm{rpm}$ for $5 \mathrm{~min}$. The plasma was aspirated into a new tube and subjected to recentrifugation at $8000 \mathrm{rpm}$ for $10 \mathrm{~min}$. Most of the plasma was removed and approximately $100-200 \mu \mathrm{L}$ was left and stored at $-70^{\circ} \mathrm{C}$ until further use. The blood samples were collected from four different hospitals in the city of Makkah.

2.2. Primers. The consensus primer pairs against dengue viruses D1 and D2 were designed using the Primer Explorer V4. The criteria for designing the serotype-specific primers (TS1, TS2, TS3, and DEN4) were based on the maximum homology of the primer pairs to the four DENV serotypes, high melting temperature, and nonhomology to the other regions of the dengue virus genome. All the oligonucleotides were commercially synthesized by Bioneer Corp., Korea, as described by Khawsak et al. [15] (Table 1).

2.3. RNA Extraction. The plasma samples of 25 suspectedcase patients were subjected to RNA isolation by QIAamp viral RNA preparation kit (Qiagen, Heldin, Germany) as per the manufacturer's instructions; initially $560 \mu \mathrm{L}$ of the AVL buffer containing the carrier RNA was pipetted into a $1.5 \mathrm{~mL}$ microcentrifuge tube. A volume of $140 \mu \mathrm{L}$ of plasma sample was added to the buffer. The contents of the tube were mixed by pulse-vortex for $15 \mathrm{~s}$ and incubated at room temperature $\left(25^{\circ} \mathrm{C}\right)$ for $10 \mathrm{~min}$. The virus particles lysis was completed within $10 \mathrm{~min}$ at room temperature. The samples were briefly centrifuged to remove the drops from the inside of the lid. An aliquot of $560 \mu \mathrm{L}$ of absolute ethanol was added to the sample and mixed by pulse-vortex for $15 \mathrm{~s}$. After mixing, the sample was centrifuged briefly to settle down the remaining drops in the inside of the lid. To ensure efficient binding, the samples were mixed thoroughly with ethanol to yield a
TABLE 1: The oligonucleotide primers used in RT-PCR (first and second strand).

\begin{tabular}{ll}
\hline Primer & Primer sequence $\left(5^{\prime}\right.$ to $\left.3^{\prime}\right)$ \\
\hline D1 & TCAATATGCTGAAACGCGCGAGAAACCG \\
D2 & TTGCACCAACAGTCAATGTCTTCAGGTTC \\
TS1 & CGTCTCAGTGATCCGGGGG \\
TS2 & CGCCACAAGGGCCATGAACAG \\
TS3 & TAACATCATCATGAGACAGAGC \\
DEN4 & TGTTGTCTTAAACAAGAGAGGTC \\
\hline
\end{tabular}

homogeneous solution. Carefully, $630 \mu \mathrm{L}$ of the solution was applied to the QIAamp Mini column without wetting the rim, followed by centrifugation at $8000 \mathrm{rpm}$ for $1 \mathrm{~min}$. The QIAamp Mini column was placed into a clean $2.0 \mathrm{~mL}$ collection tube (provided with the kit), and the tube containing the filtrate was discarded. The samples with volumes greater than $140 \mu \mathrm{L}$ were repeated for this step until all of the lysate was loaded onto the spin column. A volume of $500 \mu \mathrm{L}$ of AW1 buffer was added to the column and centrifuged at $8000 \mathrm{rpm}$ for $1 \mathrm{~min}$. The QIAamp Mini column was placed into a clean $2.0 \mathrm{~mL}$ collection tube, and the tube containing the filtrate was discarded. An aliquot of $500 \mu \mathrm{L}$ of AW2 buffer was added to the spin column, and the column was centrifuged at full speed (14,000 rpm) for $3 \mathrm{~min}$. The QIAamp Mini column was placed in a new $2.0 \mathrm{~mL}$ collection tube, and the old collection tube with the filtrate was discarded, followed by centrifugation at full speed for $1 \mathrm{~min}$. The QIAamp Mini column was placed in a clean $1.5 \mathrm{~mL}$ microcentrifuge tube. The old collection tube containing the filtrate was discarded. The AVE buffer $(60 \mu \mathrm{L})$ was applied to the column. The cap was closed and incubated at room temperature for $3 \mathrm{~min}$, followed by the last spin at $8000 \mathrm{rpm}$ for $1 \mathrm{~min}$ for the elution of viral RNA.

\subsection{Estimation of Quantity and Quality of Isolated RNA.} The RNA concentrations of the 25 RNA samples that were isolated from $25 \mathrm{DF}$ suspected patients were assessed using the NanoDrop ${ }^{\circledR}$ ND-1000 UV-Vis Spectrophotometer, which enables highly accurate analyses of extremely small samples with remarkable reproducibility. The sample retention system eliminates the need for cuvettes and capillaries, thereby allowing the estimation of viral RNA in a very low samples volume.

2.5. RT-PCR Amplification of Dengue Virus RNA (First Step). Dengue virus RNA was first converted to a cDNA by the SuperScript III First-Strand Synthesis System (Invitrogen, Carlsbad, CA, USA) prior to the PCR. The cDNA was synthesized in a $0.2 \mathrm{~mL}$ RNAse-free microtube, starting with $5.0 \mu \mathrm{L}$ of viral RNA. The RNA samples were mixed with D1 and D2 synthetic oligonucleotide primers $(0.5 \mu \mathrm{L}$ each), $1.0 \mu \mathrm{L}$ of $10 \mathrm{mM}$ dNTP mix, and $3.0 \mu \mathrm{L}$ of DEPC-treated water, for a final volume of $10 \mu \mathrm{L}$. This was incubated at $65^{\circ} \mathrm{C}$ for $5 \mathrm{~min}$ in the Applied Biosystems 96 Well Thermal Cycler to denature the RNA secondary structure and then placed on ice for $5 \mathrm{~min}$. Next, $2.0 \mu \mathrm{L} 10 \mathrm{x}$ RT buffer (Invitrogen), $4.0 \mu \mathrm{L}$ of $25 \mathrm{mM} \mathrm{MgCl}_{2}, 2.0 \mu \mathrm{L}$ 0.1 M DTT, $1.0 \mu \mathrm{L}$ RNase OUT $(40 \mathrm{U} / \mu \mathrm{L})$, and $1.0 \mu \mathrm{L}$ Superscript III RT enzyme $(200 \mathrm{U} / \mu \mathrm{L})$ were mixed in a separate RNAse-free $0.2 \mathrm{~mL}$ microtube and 
added to the RNA mixture on ice. This was incubated at $50^{\circ} \mathrm{C}$ for $50 \mathrm{~min}$ for $\mathrm{CDNA}$ synthesis and then at $85^{\circ} \mathrm{C}$ for $5 \mathrm{~min}$ to terminate the reactions in the Applied Biosystems 96 Well Thermal Cycler. The cDNA product was then chilled on ice for $5 \mathrm{~min}$, and $1.0 \mu \mathrm{L}$ of RNase $\mathrm{H}$ was added to the tube and incubated at $37^{\circ} \mathrm{C}$ for $20 \mathrm{~min}$ to degrade the viral and carrier RNA. First-strand cDNA products were stored at $-80^{\circ} \mathrm{C}$ until further use. PCR amplification was performed using primer pairs (forward dengue virus consensus primer D1 and reverse consensus primer D2), which was designed against the consensus cDNA sequence among the four dengue virus serotypes. The PCR product obtained from the RT-PCR amplification using D1 and D2 as primers was subsequently used as the template for PCR-based detection of four different DENV serotypes.

2.6. Multiplex PCR Amplification with Serotype-Specific Primers (Second Step). The PCR amplification reaction was performed to identify the dengue virus serotype in the test samples. The reaction was conducted using the PCR products from the first step of the RT-PCR that used D1 and D2 as primers. A volume of $1.0 \mu \mathrm{L}$ of the diluted materials in the ratio of 1:100 (first RT-PCR solution to the Single Distilled $\mathrm{H}_{2} \mathrm{O}$ ) was added to the PCR mixture containing all the components described for the amplification reaction using D1 as a forward and the dengue virus type-specific oligonucleotides (TS1, TS2, TS3, and DENV4) for each serotype as a reverse primer (Table 2). The PCR mixtures were subjected to 40 cycles $\left(94^{\circ} \mathrm{C}\right.$ for $30 \mathrm{~s}, 55^{\circ} \mathrm{C}$ for $60 \mathrm{~s}$, and $72^{\circ} \mathrm{C}$ for $45 \mathrm{~s}$ ) in a thermal cycler (Applied Biosystems). An aliquot of $15 \mu \mathrm{L}$ of the PCR reaction product was analyzed by electrophoresis (1.5\% agarose gel in $1 \mathrm{x}$ TBE buffer). The size of the resulting bands was characterized for each dengue virus serotype with a 100 bp DNA ladder (GeneDireX, Taiwan).

\section{Results}

The RT-PCR amplification of dengue virus RNA using D1 and D2 primer pair showed amplification of a $511 \mathrm{bp}$ RNA fragment in the case of all the serotypes of dengue virus. The $511 \mathrm{bp}$ RT-PCR product was used as a template for cDNA synthesis in the second step of RT-PCR, in which a single-tube multiplex PCR was performed using a set of serotype-specific primer pairs (D1, TS1, TS2, and DEN4). The primers used in the multiplex PCR showed highly specific amplification of viral RNA for each serotype with no nonspecific amplification of other non-DENV DNA, for example, human DNA target. Of the 25 blood samples analyzed in the present work, only six (24\%) came out positive for DENV infection. The RT-PCR and a single-tube multiplex PCR were used to analyze the plasma specimens obtained from the $25 \mathrm{DF}$ suspected patients in the city of Makkah, Western region of Saudi Arabia. Our results showed that, among the samples tested in this study, DENV-1 serotype was the most prevalent among the six DENV-positive samples (detected in three of six samples, 50\%, Figure 1(a)). The occurrence of DENV-2 serotype (two of six samples, 33.3\%, Figure 1(b)) was higher than DENV-3 serotype (one of six samples, $16.6 \%$, Figure 1(c)) among the samples positive for
TABLE 2: Dengue virus specific primers and their expected product size.

\begin{tabular}{lcc}
\hline Serotype & Primer pair & $\begin{array}{c}\text { Size of PCR } \\
\text { products (bp) }\end{array}$ \\
\hline $\begin{array}{l}\text { First step: RT-PCR } \\
\text { All serotypes }\end{array}$ & D1 + D2 & 511 \\
Second step: serotype detection & & \\
DENV-1 & D1 + TS1 & 482 \\
DENV-2 & D1 + TS2 & 119 \\
DENV-3 & D1 + TS3 & 290 \\
DENV-4 & D1 + DEN4 & 392 \\
\hline
\end{tabular}

dengue infection, whereas none of the samples demonstrated positivity for the DENV-4 serotype (Figure 1(d)).

\section{Discussion}

Dengue fever remains a serious health concern that has reemerged in the past ten years and affected thousands of individuals worldwide, especially those dwelling in the tropical and subtropical regions. Saudi Arabia, particularly the Western province, that is, Jeddah, Makkah, was declared as a DF-endemic area since the first outbreak in Jeddah in the year 1994. Our results continue to demonstrate the prevalence of dengue virus serotypes within the Western region of Saudi Arabia, particularly in the city of Makkah.

To the best of our knowledge, it is the first study identifying the highest prevalence of DENV-1 serotype in the patients with DF manifestations in Makkah city. In a study conducted in Jeddah, it was documented that the percentage of DENV-2 infection in DF-case patients is the highest compared to the DENV-1 or the DENV-3, with a dominance of the DENV-1 over the DENV-3 serotype [8]. However, in the present study, DENV-1 (50\%) was found to be the most prevalent serotype that causes DF as compared to the DENV-2 (33.3\%) or the DENV-3 (16.6\%) serotypes. In a previous study by Khan et al. [10] higher prevalence of the DENV-2 serotype was reported in contrast to the DENV-3 in DF cases in Makkah, with no DENV-1 detection. DENV1 appears to be the predominant dengue virus serotype in various regions, as it was the frequently detected DENV serotype in DF/DHF/DSS cases in the endemic areas of the Middle East, Asia, South America, or even in the nondengue endemic countries, such as Japan, where DENV is introduced by the travelers coming from the endemic regions [6, 16-18]. On the whole, it appears that DENV-3 has least circulation not only in the cases tested in current study, but also in the other patients belonging to the different areas in Saudi Arabia $[7,8,10,19]$ and elsewhere [20]. DENV-4 neither was detected in our study, nor has been detected in other investigations done so far on serological or molecular typing of dengue virus in Saudi Arabia [7, 8, 10, 19] or the other neighboring countries [20, 21]. Overall, DENV-1, DENV-2, and DENV-3 have been the predominant serotypes of dengue virus in the Middle East, particularly Saudi Arabia and Yemen [3], which is further confirmed by this study. 


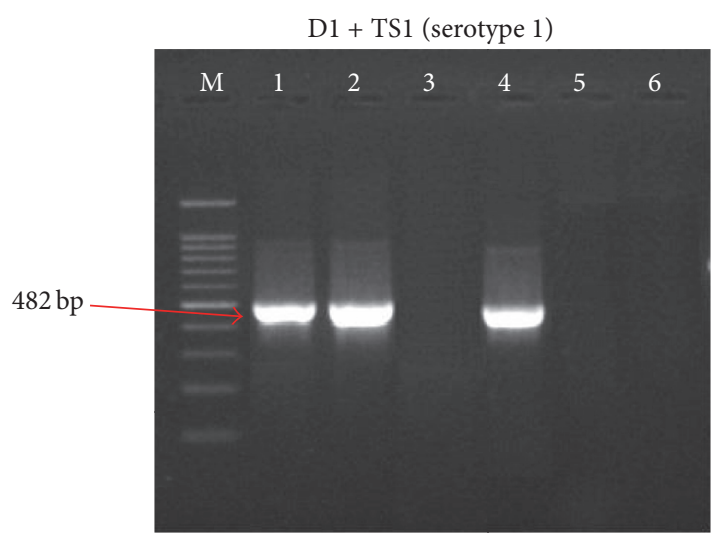

(a)

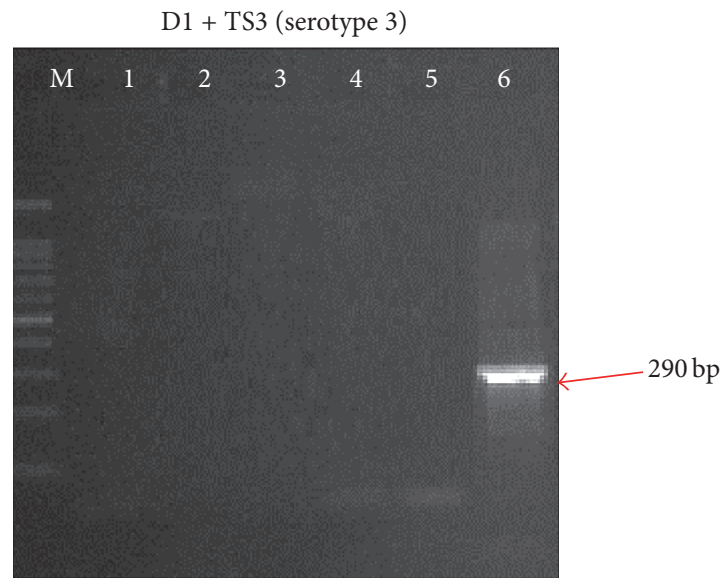

(c)

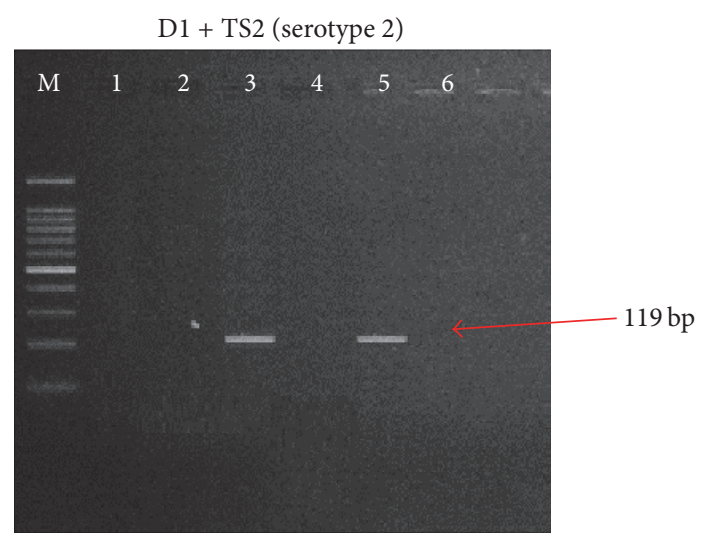

(b)

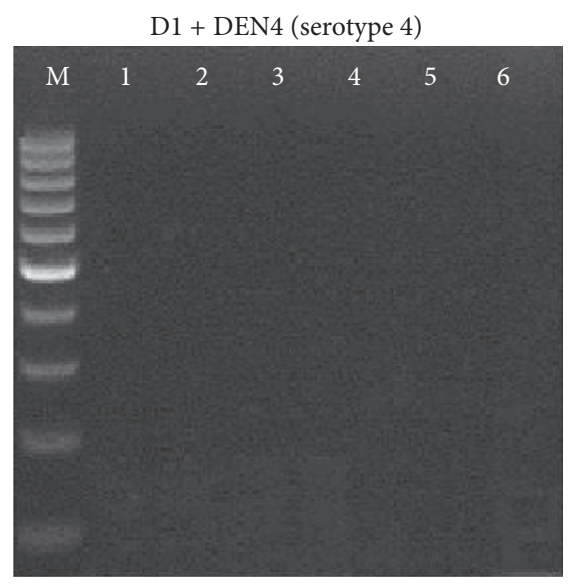

(d)

FIGURE 1: Identification of dengue virus serotypes by the specific primers. Four sets of specific primers were (a) D1 and TS1 for DENV-1, (b) D1 and TS2 for DENV-2, (c) D1 and TS3 for DENV-3, and (d) D1 and DEN4 for DENV-4.

Although the incidence of dengue fever in Saudi Arabia has increased in the past three years (6512 cases in $2013 ; 2081$ cases in 2014; 4312 cases in 2015) [22], the low prevalence of DENV detected in this study (24\%) may be attributed, in part, to different factors. The total number of DF cases recorded in Saudi Arabia in the year 2015 was 4312, of which only $855(19.8 \%)$ cases were recorded in Makkah, as compared to $3161(73.3 \%)$ cases in Jeddah [22]. Most of these cases were recorded during the months from May through July, whereas in our study we analyzed the samples collected in the months other than the above. Overall, the prevalence of DENV in Saudi Arabia, as reported in different studies, ranged from $31.7 \%$ to $56.9 \%$ [13]. The other reason for such low prevalence of DENV infection in the present study might be the stage of disease or the day of sample collection after onset of the illness. It has been reported that the best period for DENV RNA detection in blood samples is in the acute phase of infection, that is, the first five days after onset of the symptoms [7], but, here, we did not know the stage of infection wherein the patients were sampled; thus, it is possible that those six positive samples were withdrawn from patients who were in the acute phase of infection. It is also worth noting that the negative samples might be of the patients who may not actually had DENV infection, in spite of presenting with dengue-like symptoms, the reason being that most of the hospitals in Saudi Arabia diagnose dengue virus infection by estimation of blood parameters, particularly, platelet counts which is only a predictive parameter in case of dengue fever [23].

\section{Conclusion}

Our results confirm the circulation of DENV-1, DENV-2, and DENV-3 in the city of Makkah, in the Western region of Saudi Arabia. The present work highlights the significance of continuous surveillance of DENV serotypes in Makkah, as annually during pilgrimage (Hajj) and the minor pilgrimage (Umrah), more than 3 million people visit the city from different geographic locations including areas that are DFendemic zones. Novel serotypes, like DENV-4 and DENV-5, may emerge in the city of the entire region by the travelers (who wish to perform religious rituals) or the locals who travel to dengue endemic areas for vacation and holidays. Thus, it is highly imperative to discriminate the local origin serotypes from the traveler-introduced serotypes, for which continued surveillance is vital. 


\section{Competing Interests}

The authors declare that there are no competing interests regarding the publication of this paper.

\section{Acknowledgments}

The authors would like to express their gratitude to all those who helped them to obtain blood samples of denguesuspected patients. This project was fully funded by Umm Al-Qura Institute of Scientific Research and the Revival of Islamic Heritage (Grant no. 43405055).

\section{References}

[1] D. J. Gubler, "Epidemic dengue/dengue haemorrhagic fever: a global public health problem in the 21st century," Dengue Bulletin, vol. 21, pp. 1-14, 1997.

[2] J. M. Heilman, J. De Wolff, G. M. Beards, and B. J. Basden, "Dengue fever: a Wikipedia clinical review," Open Medicine, vol. 8, no. 4, pp. e105-e115, 2014.

[3] T. Nedjadi, S. El-Kafrawy, S. S. Sohrab, P. Desprès, G. Damanhouri, and E. Azhar, "Tackling dengue fever: current status and challenges," Virology Journal, vol. 12, 2015.

[4] N. Khetarpal and I. Khanna, "Dengue fever: causes, complications, and vaccine strategies," Journal of Immunology Research, vol. 2016, Article ID 6803098, 14 pages, 2016.

[5] J.-H. Park and D.-W. Lee, "Dengue Fever in South Korea, 20062010," Emerging Infectious Diseases, vol. 18, no. 9, pp. 1525-1527, 2012.

[6] Y. Mizuno, Y. Kato, S. Kano, and T. Takasaki, "Imported malaria and dengue fever in returned travelers in Japan from 2005 to 2010," Travel Medicine and Infectious Disease, vol. 10, no. 2, pp. 86-91, 2012.

[7] M. Fakeeh and A. M. Zaki, "Virologic and serologic surveillance for dengue fever in Jeddah, Saudi Arabia, 1994-1999," American Journal of Tropical Medicine and Hygiene, vol. 65, no. 6, pp. 764767, 2001.

[8] M. Fakeeh and A. M. Zaki, "Dengue in Jeddah, Saudi Arabia, 1994-2002," Dengue Bulletin, vol. 27, pp. 13-18, 2003.

[9] M. Ayyub, A. M. Khazindar, E. H. Lubbad, S. Barlas, A. Y. Alfi, and S. Al-Ukaily, "Characteristics of dengue fever in a large public hospital, Jeddah, Saudi Arabia," Journal of Ayub Medical College Abbottabad, vol. 18, pp. 9-13, 2006.

[10] N. A. Khan, E. I. Azhar, S. El-Fiky et al., "Clinical profile and outcome of hospitalized patients during first outbreak of dengue in Makkah, Saudi Arabia," Acta Tropica, vol. 105, no. 1, pp. 3944, 2008.

[11] T. A. Al-Azraqi, A. A. El Mekki, and A. A. Mahfouz, "Seroprevalence of dengue virus infection in Aseer and Jizan regions, Southwestern Saudi Arabia," Transactions of the Royal Society of Tropical Medicine and Hygiene, vol. 107, no. 6, pp. 368-371, 2013.

[12] S. A. El-Kafrawy, S. S. Sohrab, S. A. Ela et al., "Multiple introductions of dengue 2 virus strains into Saudi Arabia from 1992 to 2014," Vector-Borne and Zoonotic Diseases, vol. 16, no. 6, pp. 391-399, 2016.

[13] A. Alhaeli, S. Bahkali, A. Ali, M. S. Househ, and A. A. ElMetwally, "The epidemiology of Dengue fever in Saudi Arabia: a systematic review," Journal of Infection and Public Health, vol. 9, no. 2, pp. 117-124, 2016.
[14] W. Shahin, A. Nassar, M. Kalkattawi, and H. Bokhari, "Dengue fever in a tertiary hospital in Makkah, Saudi Arabia," Dengue Bulletin, vol. 33, no. 1, pp. 34-43, 2009.

[15] P. Khawsak, S. Phantana, and K. Chansiri, "Determination of dengue virus serotypes in Thailand using PCR based method," Southeast Asian Journal of Tropical Medicine and Public Health, vol. 34, no. 4, pp. 781-785, 2003.

[16] A. Tuiskunen, V. Monteil, S. Plumet et al., "Phenotypic and genotypic characterization of dengue virus isolates differentiates dengue fever and dengue hemorrhagic fever from dengue shock syndrome," Archives of Virology, vol. 156, no. 11, pp. 2023 2032, 2011.

[17] M. G. Teixeira, "Few characteristics of dengue's fever epidemiology in Brazil," Revista do Instituto de Medicina Tropical de Sao Paulo, vol. 54, S18, pp. S1-S4, 2012.

[18] L. Jiang, X. Wu, Y. Wu et al., "Molecular epidemiological and virological study of dengue virus infections in Guangzhou, China, during 2001-2010," Virology Journal, vol. 10, 2013.

[19] A. A. El-Badry, H. A. El-Beshbishy, K. H. Al-Ali, A. M. AlHejin, and W. S. M. El-Sayed, "Molecular and seroprevalence of imported dengue virus infection in Al-Madinah, Saudi Arabia," Comparative Clinical Pathology, vol. 23, no. 4, pp. 861-868, 2014.

[20] T. A. Madani, E.-T. M. E. Abuelzein, H. M. S. Al-Bar et al., "Outbreak of viral hemorrhagic fever caused by dengue virus type 3 in Al-Mukalla, Yemen," BMC Infectious Diseases, vol. 13, article 136, 2013.

[21] L. Vinner, C. Domingo, A.-C. B. Ostby, K. Rosenberg, and A. Fomsgaard, "Cases of travel-acquired dengue fever in Denmark 2001-2009," Clinical Microbiology and Infection, vol. 18, no. 2, pp. 171-176, 2012.

[22] Ministry of Health; Department of Statistics, Health Statistical Year Book 2016, Saudi Ministry of Health, Riyadh, Saudi Arabia, 2016.

[23] K. Jayashree, G. C. Manasa, P. Pallavi, and G. V. Manjunath, "Evaluation of platelets as predictive parameters in dengue fever," Indian Journal of Hematology and Blood Transfusion, vol. 27, no. 3, pp. 127-130, 2011. 


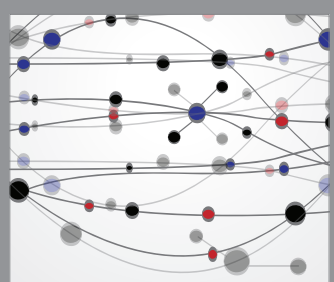

The Scientific World Journal
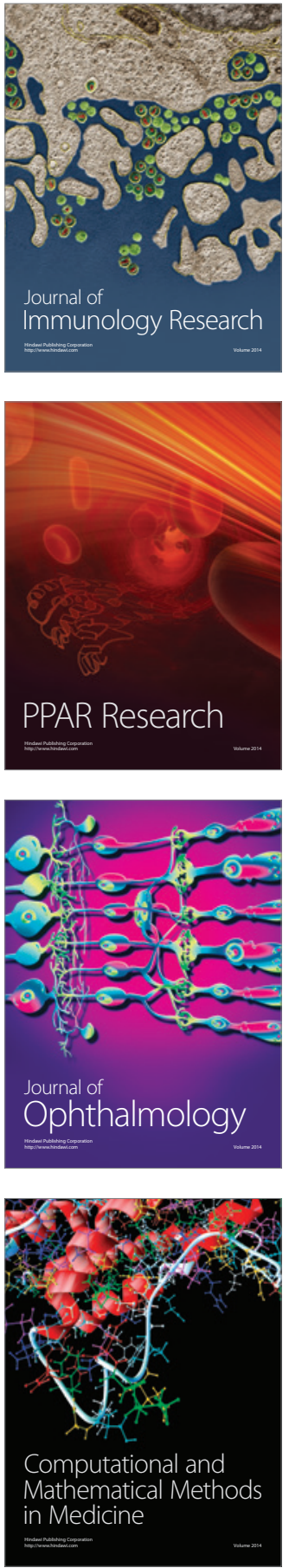

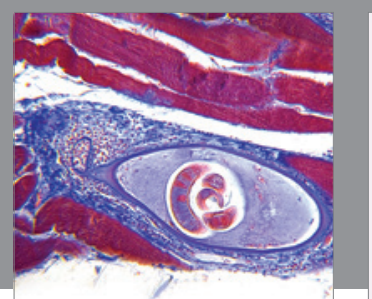

Gastroenterology Research and Practice
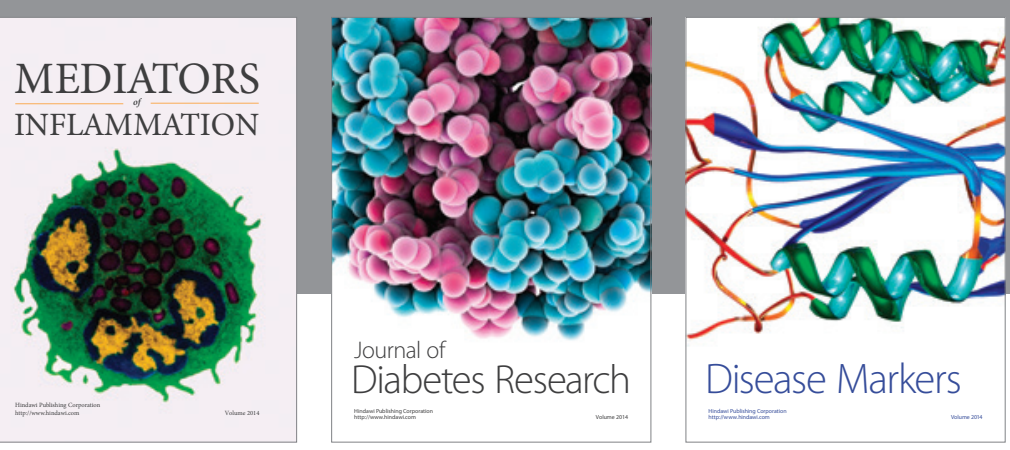

Disease Markers

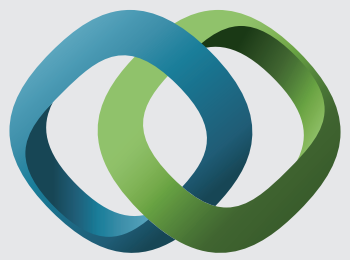

\section{Hindawi}

Submit your manuscripts at

https://www.hindawi.com
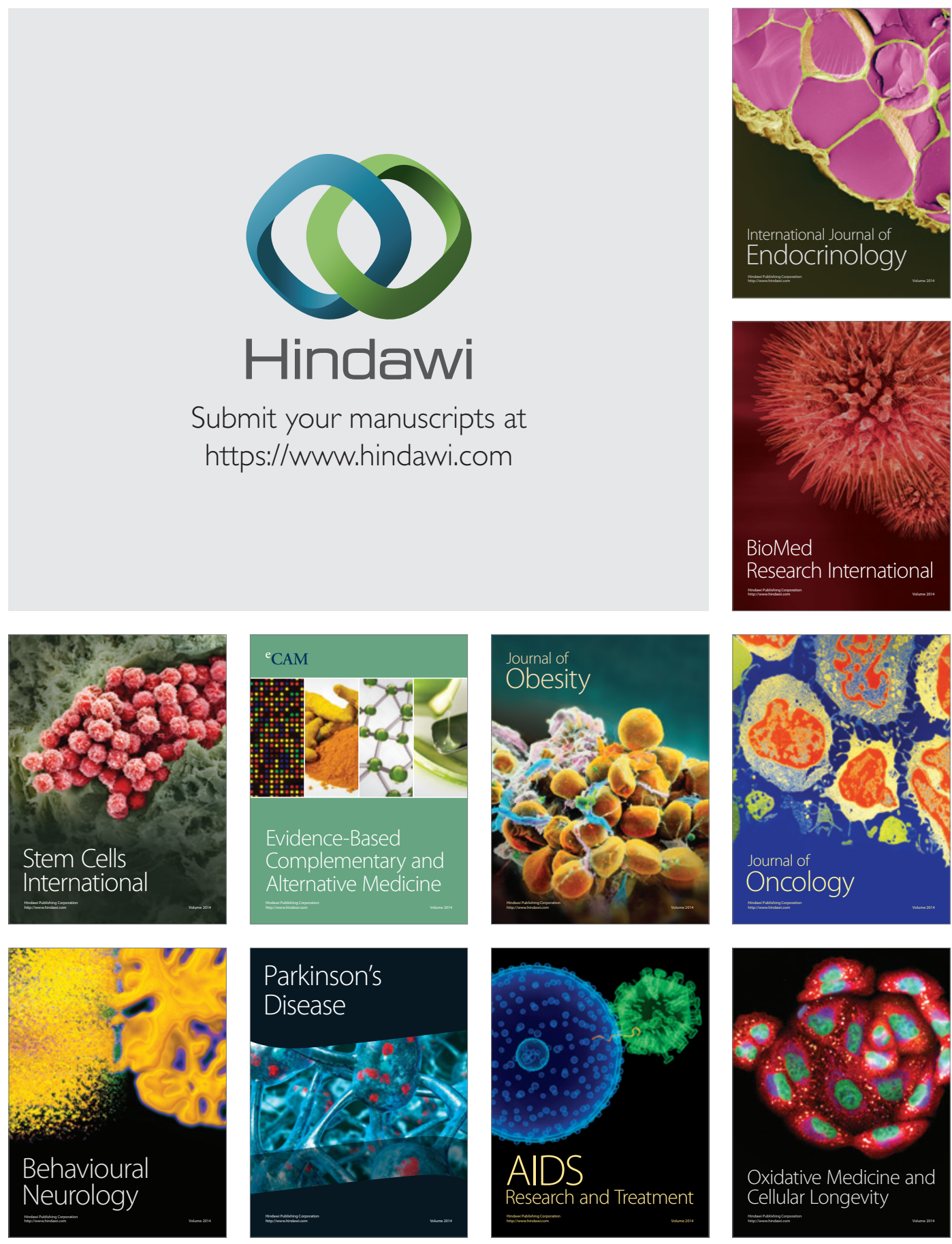